\title{
Photoproduction of Pion Pairs with High Invariant Mass
}

\author{
H. Alvensleben, U. J. Becker, William K. Bertram, M. Chen, K. J. Cohen, \\ R. T. Edwards, T. M. Knasel, R. Marshall, D. J. Quinn, M. Rohde, \\ G. H. Sanders, H. Schubel, and Samuel C. C. Ting \\ Deutsches Elektronen-Synchrotron DESY, Hamburg, Germany, and Department of Physics and Laboratory for \\ Nuclear Science, Massachusetts Institute of Technology, Cambridge, Massachusetts 02139
}

(Received 24 November 1970)

\begin{abstract}
We have measured the yield of $\pi^{+} \pi^{-}$pairs from the reaction $\gamma+\mathrm{C} \rightarrow \mathrm{C}+\pi^{+}+\pi^{-}$in the invariant-mass region $0.6<m<1.8 \mathrm{GeV} / c^{2}$. The result, based on $10^{5}$ events, shows that the data, at high energy and very small momentum transfer, exhibit an enhancement in the region $1.3<m<1.8 \mathrm{GeV} / c^{2}$.
\end{abstract}

We have measured the cross section for production of pion pairs in the reaction

$$
\gamma+\mathrm{C} \rightarrow \mathrm{C}+\pi^{+}+\pi^{-}
$$

to study in detail the production mechanism and to search for resonances with quantum numbers $J^{p}=0^{+}, 1^{-}, 2^{+}, \cdots$ decaying into $\pi^{+} \pi^{-}$pairs. There are many theoretical reasons for interest in the existence of new vector mesons. Examples are the following: (1) The validity of the vector-dominance model $^{1}$ depends on whether there are other vector mesons beyond $\rho, \omega, \varphi$. (2) The quark model $^{2}$ allows the existence of a new nonet of vector mesons with the $q \bar{q}$ state ${ }^{3} D_{1}$ in addition to the known $\rho, \omega, \varphi$, which correspond to the ${ }^{3} S_{1}$ state. (3) The Veneziano model $^{3}$ predicts the existence of a meson with mass around $1400 \mathrm{MeV} /$ $c^{2}$ which could decay into a $\pi^{+} \pi^{-}$final state.

The experiment was carried out at the $7.5-\mathrm{GeV}$ DESY electron synchrotron. Pion pairs were produced by letting a bremsstrahlung beam strike a $3-\mathrm{cm}$ carbon target, and detected by a pair of large-aperture magnetic spectrometers. ${ }^{4}$ The mass range from 0.6 to $1.8 \mathrm{GeV} / c^{2}$ was covered by taking a series of fifteen measurements with opening angles from $11^{\circ}$ to $30^{\circ}$, keeping the mean momenta accepted by each spectrometer arm constant.

The following properties of the symmetric pair spectrometer were essential for this experiment:

(1) The acceptance of each spectrometer was defined by scintillation counters that did not optically see the target. The random accidental rates were always $<3 \%$ of the true coincidence rates.

(2) The spectrometer recombined rays of constant $p_{ \pm} \theta_{ \pm} \propto m$, where $p_{ \pm}$and $\theta_{ \pm}$are the momentum and angle of the particle passing through each spectrometer arm. It had a large acceptance in $p_{ \pm}$and $\theta_{ \pm}$, a mass resolution of $\pm 15 \mathrm{MeV} / c^{2}$, a momentum resolution of $\pm 150 \mathrm{MeV} / c$, and a $t_{\perp}$ resolution of $\pm 0.001(\mathrm{GeV} / c)^{2}$.

(3) Two large-aperture threshold Cherenkov counters were used in each arm to reject particles heavier than pions. The efficiency for counting pions was better than $99 \%$ and nearly independent of the spectrometer setting. To keep inelastic contributions to Reaction (1) constant and as small as possible, all the data were collected with photon energy $k$ close to the peak energy $k_{\text {max }}\left(k / k_{\text {max }}=0.9\right)$.

A total of $10^{5}$ events were detected. The data were corrected for small systematic effects such as beam attenuation in the target, target out, nuclear absorption of pions in the target and counters, dead time, accidentals, etc. All these corrections were checked by measurement in the same spectrometer to be accurate to $\approx 1 \%$.

The acceptance was calculated with a Monte Carlo method incorporating the effects of multiple scattering and $\pi$ decay in flight along the spectrometer. A sufficient number of Monte Carlo events were treated so that the errors in the measured cross sections are due only to experimental statistics and small systematic uncertainties. In calculating the cross section $d^{2} \sigma / d \Omega d m$ a decay angular distribution of $(3 / 8 \pi) \sin ^{2} \theta^{*}$ in the helicity frame was assumed.

As there is no adequate description for the production of resonances of strongly interacting particles and no precise information on the nuclear physics of carbon available, we have grouped the data in a three-dimensional matrix $d^{2} \sigma\left(m, p, t_{\perp}\right)$ $/ d \Omega d m$, where $p$ is the total di-pion momentum in the laboratory, $m$ is the invariant mass of the pion pair, and $t_{\perp}$ is the squared transverse momentum transfer to the recoil nucleus. In this way the behavior of the production cross section as a function of $m, p$, and $t_{\perp}$ is preserved independent of any theoretical assumptions. The cross-section matrix $d^{2} \sigma\left(m, p, t_{\perp}\right) / d \Omega d m$ has dimensions of $(40,5,20)$, where the bin sizes are 


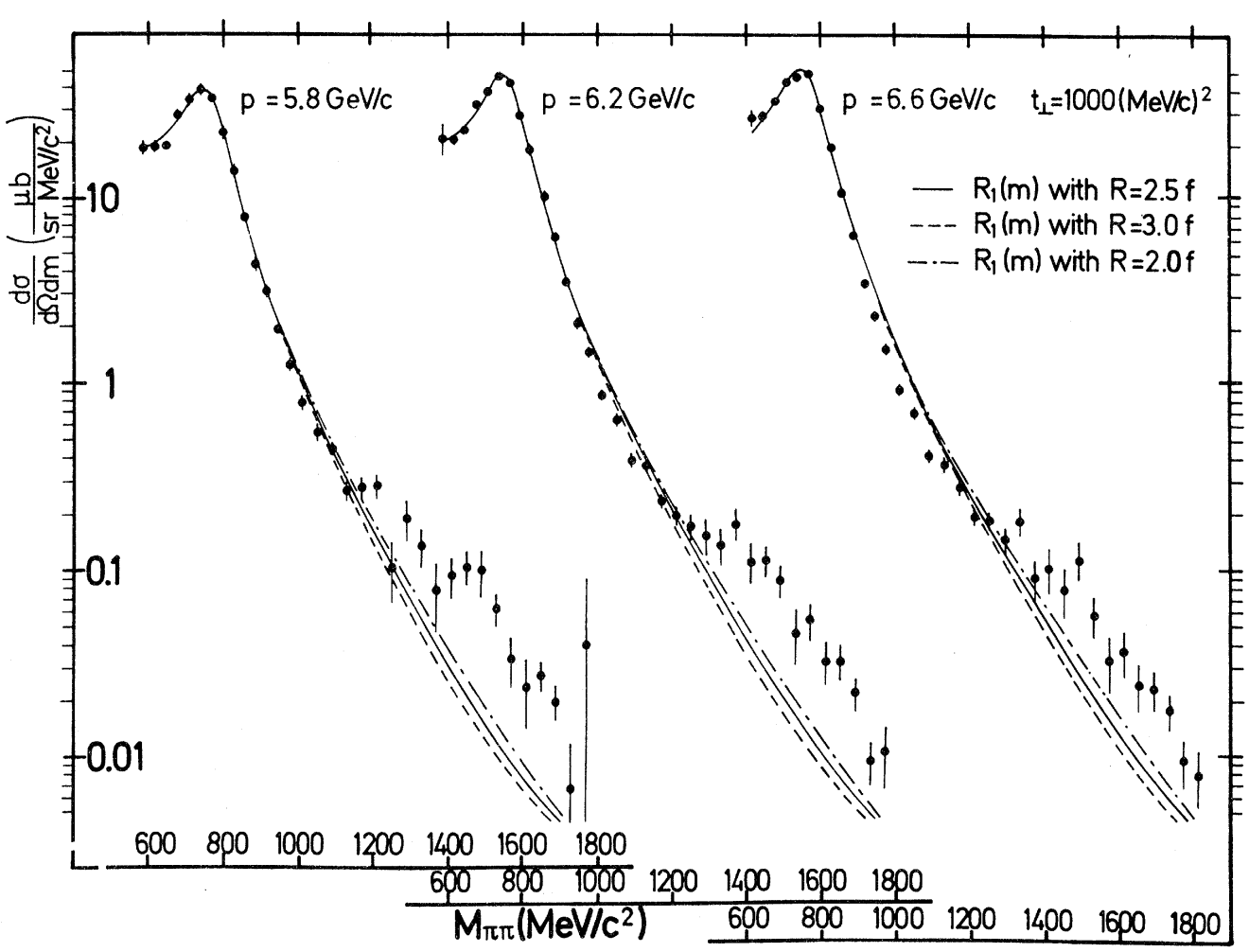

FIG. 1. Projection of the cross-section matrix on the $(m, p)$ plane. For masses higher than $1.0 \mathrm{GeV} / \mathrm{c}^{2} \mathrm{the}$ bin size was changed to $40 \mathrm{MeV} / \mathrm{c}^{2}$. The curves are fits (see text) which were simultaneously applied to the mass region $0.6<m<0.9 \mathrm{GeV} / c^{2}$, momentum $5.4<p<6.8 \mathrm{GeV} / c$, and transverse momentum transfer $0<t_{\perp}<0.01(\mathrm{GeV} / c)^{2}$. To keep inelastic contributions constant and small, all the data were collected with photon energy $k$ close to the peak energy $\boldsymbol{k}_{\max }$.

$30 \mathrm{MeV} / c^{2}$ in $m, 400 \mathrm{MeV} / c$ in $p$, and 2000 $\mathrm{MeV}^{2} / c^{2}$ in $t_{\perp}$.

Figure 1 shows a typical cross section as a function of $m$ and $p$ for a fixed $t_{\perp}=0.001(\mathrm{GeV} / c)^{2}$. Figure 2 shows the behavior of the cross section as a function of $m$ and $t_{\perp}$ for fixed $p=6.2 \mathrm{GeV} / c$. These figures contain only a small part of the data. The measured cross section exhibits the following characteristics:

(1) Independent of the $p$ and $t_{\perp}$ intervals chosen, the spectra decrease by about 4 orders of magnitude from $m=m_{\rho}$ to $m=1.8 \mathrm{GeV} / c^{2}$. At $m \approx m_{\rho}$ the cross section agrees with previous measurements. ${ }^{4}$
(2) Independent of the $p$ interval considered, the cross sections decrease with $t_{\perp}$ as $\exp \left[A(m)^{*} t_{\perp}\right]$. At the mass of the $\rho$ we have $A\left(m_{\rho}\right) \approx 50(\mathrm{GeV} / c)^{-2}$. After subtraction of background $A\left(m_{\rho}\right) \approx 70(\mathrm{GeV} /$ $c)^{-2}$. For $m>1.1 \mathrm{GeV} / c^{2}$ a steady decrease in slope is noticed with $A(1.6) \approx 25(\mathrm{GeV} / c)^{-2}$.

(3) No statistically significant narrow enhancement is observed. However, a broad enhancement in the mass region $1.3<m<1.8 \mathrm{GeV} / c^{2}$ is seen.

In the mass region $0.6<m<0.9 \mathrm{GeV} / c^{2}$, where the spectra are dominated by $\rho$ production, the data were analyzed by fitting with the following equation:

$$
\begin{aligned}
& \frac{d^{2} \sigma}{d \Omega d m}\left(m, p, t_{\perp}\right)=\frac{p^{2}}{\pi} 2 m R_{n}(m) \operatorname{closure}\left(t_{\|}, t_{\perp}, R, \sigma_{\rho N}, \beta\right)+f_{\mathrm{BG}}(m), \\
& R_{1}(m)=\left(\frac{m_{\rho}}{m}\right)^{4} r(m), \quad r(m)=\frac{1}{\pi} \frac{m_{\rho} \Gamma_{\rho}}{\left(m_{\rho}^{2}-m^{2}\right)^{2}+m_{\rho}^{2} \Gamma_{\rho}^{2}}, \quad \Gamma_{\rho}=\frac{m_{\rho}}{m}\left(\frac{m^{2}-4 m_{\pi}^{2}}{m_{\rho}^{2}-4 m_{\pi}^{2}}\right)^{3 / 2} \Gamma_{0}, \\
& R_{2}(m)=R_{1}(m), \quad \Gamma_{\rho}=\Gamma_{0}, \quad R_{3}(m)=r(m), \quad \Gamma_{\rho}=\Gamma_{0}, \quad f_{\mathrm{BG}}(m)=\sum_{i=1}^{3} a_{i} m^{i-1},
\end{aligned}
$$




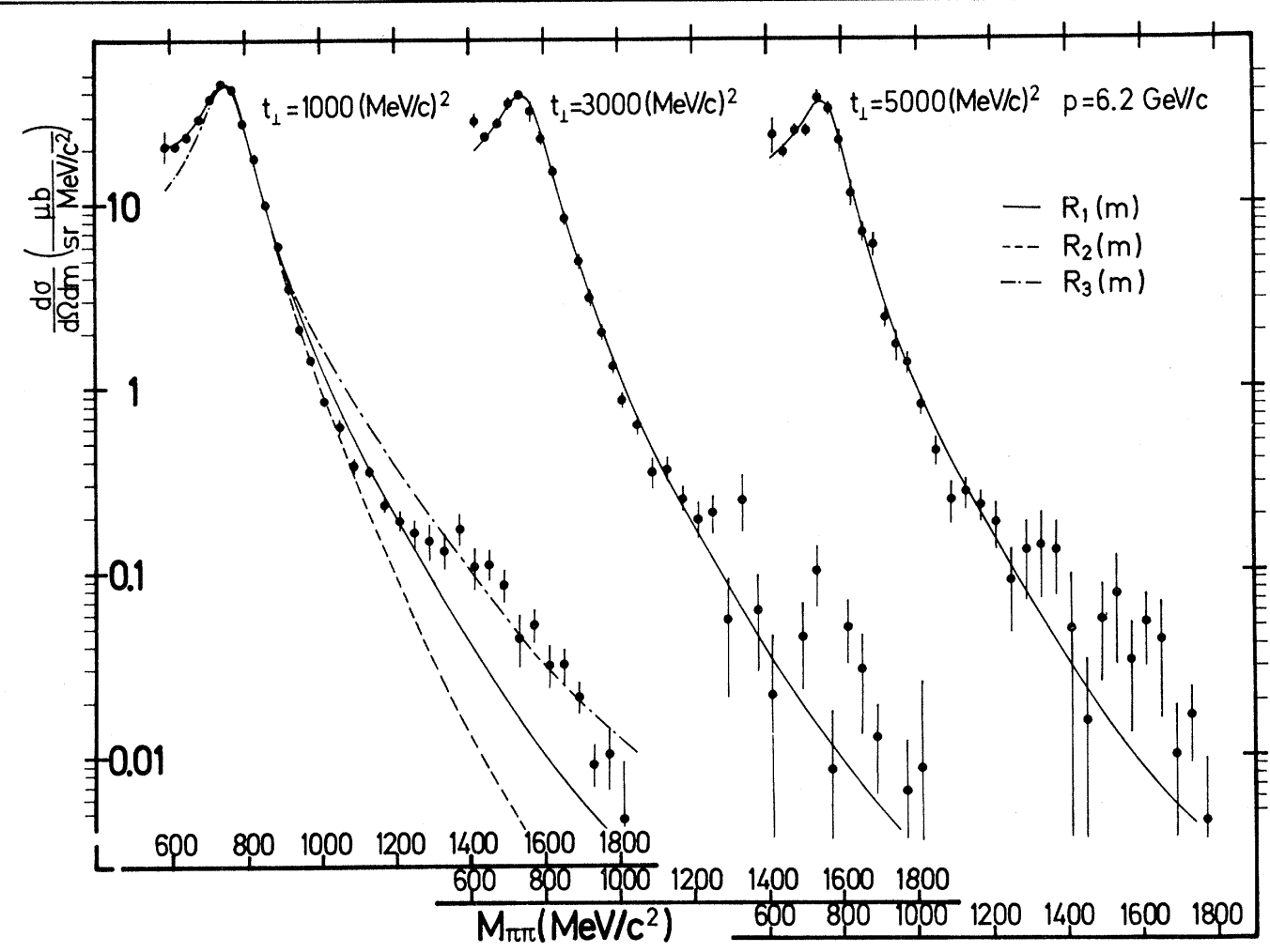

FIG. 2. Projection of the cross-section matrix on the $\left(m, t_{\llcorner}\right)$plane. The curves are fits (see text) applied to the region above.

$R_{1}(m)$ represents the fitting of the di-pion spectrum to a $\rho$-production term, where the relativistic $p$-wave resonance formula $r(m)$ is modified by the phenomenological Ross-Stodolsky ${ }^{5}$ term $\left(m_{\rho} / m\right)^{4}$ plus a general background. $R_{2}(m)$ is $R_{1}(m)$ with constant width. $R_{3}(m)$ is the normal relativistic Jackson ${ }^{6}$ formula with a constant width.

The function closure $\left(t_{\|}, t_{\perp}, R, \sigma_{\rho N}, \beta\right)$ is the forward-production cross section as calculated by Trefil ${ }^{7}$ including coherent and incoherent production. The closure function has been tested by comparison with proton-nuclei scattering at large momentum transfers and at $19 \mathrm{GeV} .^{7}$ It was found to agree well with the experimental data. This function describes the dependence of the cross section on longitudinal and transverse momentum transfer, $t_{\|}$and $t_{\perp}$, respectively. The function requires the following parameters (obtained in previous experiments) ${ }^{4,8}$ : (a) the Woods-Saxon radius $R=2.5 \mathrm{~F}$ for carbon, (b) the $\rho$-nucleon total cross section $\sigma_{\rho N}=26.7 \mathrm{mb}$, and (c) the ratio of the real-to-imaginary part of the $\rho$-nucleon scattering amplitude for $\beta=-0.2$. When approximated by the exponential $\exp [B(t$ $\left.\left.-t_{\mathrm{min}}\right)\right]$, the slope $B$ changes from $B \approx 79(\mathrm{GeV} /$ $c)^{-2}$ at $m_{\rho}$ to $\approx 25(\mathrm{GeV} / c)^{-2}$ at $1.8 \mathrm{GeV} / c^{2}$. This is in agreement with the measured change of the slope $\left[A\left(m_{\rho}\right) \approx 70, A(1.6) \approx 25 \mathrm{GeV} / c^{2}\right]$. The background function is a polynomial in $m$. Equation (2) was then compared with the measured crosssection matrix $d^{2} \sigma\left(m, p, t_{\perp}\right) / d \Omega d m$ in the region of momenta $4.8<p<6.8 \mathrm{GeV} / c$, of momentum transfers $0<t_{\perp}<0.01(\mathrm{GeV} / c)^{2}$, and masses 0.6 $<m<0.9 \mathrm{GeV} / c^{2}$.

The solid lines in Figs. 1 and 2 represent for $0.6<m<0.9 \mathrm{GeV} / c^{2}$ the best fit by Eq. (2) using $R_{1}(m)$ with an energy-dependent width $\Gamma_{\rho}(m)$. The fits are extrapolated to give the curves for $m$ $>0.9 \mathrm{GeV} / c^{2}$. The dotted lines in Fig. 1 demonstrate the effect of varying the Woods-Saxon radius by $\pm 0.5 \mathrm{~F}$. The first graph of Fig. 2 shows fits with $R_{2}(m)$ and $R_{3}(m)$ and their extrapolation beyond $0.9 \mathrm{GeV} / c^{2}$. One notices that $R_{3}(m)$ does not describe the data well. [Other forms of $R(m)$ have been tried but yield worse results than $R_{1}(m), R_{2}(m), R_{3}(m)$.]

All these fits fail to give a reasonable description of the data for $m>0.9 \mathrm{GeV} / c^{2}$. The observed excess in the mass region $1.3<m<1.8 \mathrm{Gev} / c^{2}$ cannot be explained by radius variation or simple modifications of the line shape. This enhance- 


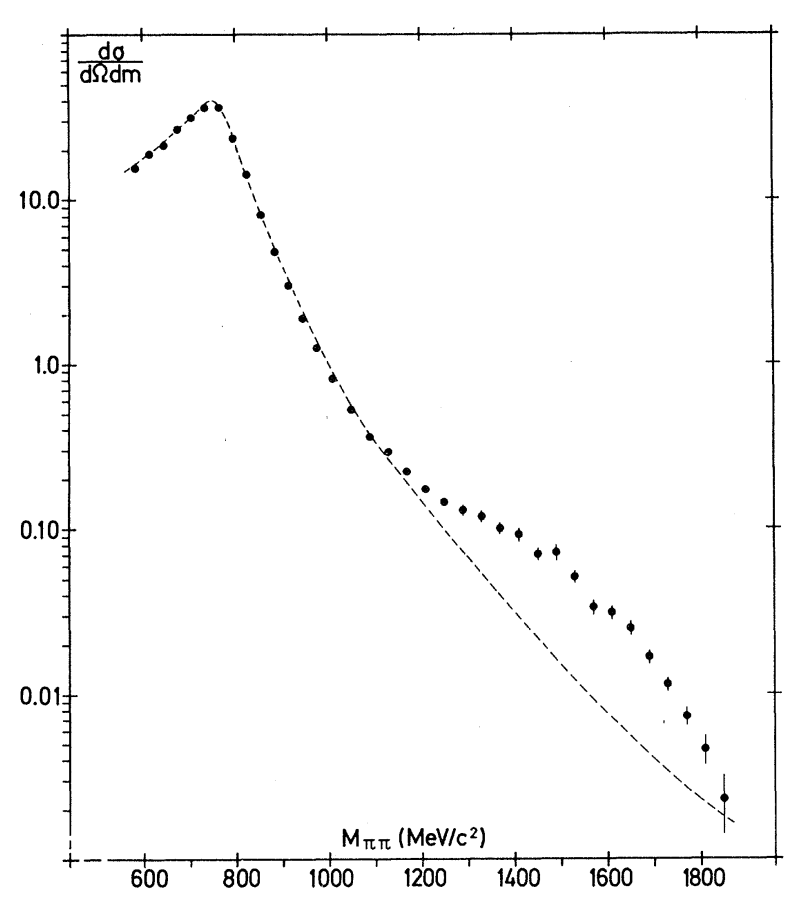

FIG. 3. Mass spectrum for all data averaged over the spectrometer aperture. Errors are statistical only. The curve is a best eye fit based on Figs. 1 and 2 .

ment cannot be easily attributed to an inelastic process since it is seen at all momentum intervals up to $k_{\mathrm{m} \text { ax }}$ and does not show a significant increase with increasing $t_{\perp}$.

Figure 3 shows all the data combined. The cross sections $d^{2} \sigma / d \Omega d m$ were averaged over the spectrometer aperture. The curve is an eye fit using Figs. 1 and 2 as a guide. Again a broad enhancement is clearly seen. No statistically significant resonances of narrow width were observed; in particular the predicted $\rho^{\prime}$ at $1.4 \mathrm{GeV} /$ $c^{2}$ was not clearly seen. ${ }^{9}$ It would be difficult to set an upper limit for other possible vector mesons since that would require knowledge of their amplitudes for decay into $\pi^{+} \pi^{-}$together with their relative phase angles when interfering with the $\rho \rightarrow \pi \pi$ events.

In conclusion, this experiment shows that the observed $\pi^{+} \pi^{-}$mass spectrum does not exhibit a clear, sharp, narrow resonance. The data do exhibit a broad enhancement in the mass region 1.3 $<m<1.8 \mathrm{GeV} / c^{2}$. This enhancement could not be easily explained by dependence on nuclear physics or decay spectra parameters. The existence of a broad resonance decaying into $\pi^{+} \pi^{-}$pairs with a proper relative phase to $\rho \rightarrow \pi \pi$ could conceivably explain the observed enhancement.

We are grateful for the support of Professor W. Jentschke, Professor V. F. Weisskopf, Professor P. Demos, Professor A. G. Hill, Professor H. Joos, and Professor H. O. Wuester, who made this collaboration possible. We wish' to thank Dr. L. Bertocchi, Dr. J. S. Trefil, Dr. B. Margolis, Dr. E. Lohrmann, Dr. W. Busza, and Dr. P. Biggs for interesting comments. We are grateful to Dr. D. Lublow and Mr. H. Kumpfert for excellent operation of the computing machine and the synchrotron, and to Miss I. Schulz, Mrs. H. Feind, and Mr. P. Berges for technical assistance.

\footnotetext{
${ }^{1}$ H. Joos, Acta. Phys. Austr., Suppl. 4, 320 (1967); J. J. Sakurai, in Lecture in Theoretical Physics, edited by K. T. Mahanthappa, W. E. Brittin, and A. O. Barut (Gordon and Breach, New York, 1969), Vol. XI A, Part 1.

${ }^{2} \mathrm{H}$. L. Lipkin, in Proceedings of the Lund International Conference on Elementary Particles, edited by G. von Dardel (Berlingska Boktryckeriet, Lund, Sweden, 1970), p. 55.

${ }^{3} \mathrm{~J}$. A. Shapiro, Phys. Rev. 179, 1345 (1969).

${ }^{4} \mathrm{H}$. Alvensleben et al., Nucl. Phys. B18, 333 (1970).

${ }^{5}$ M. Ross and L. Stodolsky, Phys. Rev. 149, 1172 (1966); G. Kramer and J. L. Uretsky, Phys. Rev. 181, 1918 (1969).

${ }^{6}$ J. D. Jackson, Nuovo Cimento 34, 1644 (1964).

${ }^{7} J$. S. Trefil, Nucl. Phys. B11, 330 (1969)。

${ }^{8} \mathrm{H}$. Alvensleben et al., Phys. Rev. Lett. 25, 1377 (1970).

${ }^{9}$ G. McClellan et al., Phys. Rev。 Lett. 23, 718 (1969), where an attempt at setting upper limits on $\rho^{\prime}$ and $\rho^{\prime \prime}$ was made. F. Bulos et al., Stanford Linear Accelerator Center Report No. SLAC PUB-751, 1970 (unpublished).
} 